\title{
Treatment with bevacizumab and FOLFOXIRI in patients with advanced colorectal cancer: presentation of two novel trials (CHARTA and PERIMAX) and review of the literature
}

Alexander Stein ${ }^{1 \dagger}$, Gabriel Glockzin ${ }^{2 \dagger}$, Andreas Wienke ${ }^{3}$, Dirk Arnold ${ }^{1}$, Thomas Edelmann ${ }^{4}$, Bert Hildebrandt ${ }^{5}$, Stephan Hollerbach ${ }^{6}$, Gerald Illerhaus ${ }^{7}$, Alfred Königsrainer ${ }^{8}$, Michael Richter ${ }^{9}$, Hans J Schlitt ${ }^{2}$ and Hans-Joachim Schmoll ${ }^{3 *}$

\begin{abstract}
Background: More than half of patients with colorectal cancer will develop metastatic disease either evident at the time of initial diagnosis or during their course of disease. Besides multidisciplinary management further treatment intensification is warranted to improve the still limited prognosis.

Methods/design: In these two multi-centre, randomized phase II trials, conducted in Germany, 380 patients with R0-resectable colorectal liver metastases (PERIMAX) and with unresectable, metastatic colorectal cancer (CHARTA) will be recruited. Patients previously untreated for metastatic disease with either synchronous or metachronous metastases are randomly assigned in a 1:1 ratio to resection of colorectal liver metastases followed by postoperative FOLFOX for 6 months or perioperative FOLFOXIRI and bevacizumab for 3 months pre- and postoperative and resection (PERIMAX), or to induction chemotherapy with FOLFOX and bevacizumab +/- irinotecan for a maximum of 6 months followed by maintenance treatment with fluoropyrimidine and bevacizumab. The primary objective of these trials is to evaluate the feasibility and efficacy of FOLFOXIRI and bevacizumab in metastatic colorectal cancer. Primary endpoint is failure free survival rate at 18 months in the PERIMAX trial and progression free survival rate at 9 months in CHARTA. Secondary objectives include efficacy, safety and tolerability.

Discussion: The CHARTA and PERIMAX trials are designed to evaluate the benefits and limitations of a highly active four-drug regimen in distinct treatment situations of metastatic CRC. Eligible patients are classified into resectable liver metastases to be randomized to perioperative treatment with FOLFOXIRI and bevacizumab or postoperative FOLFOX in the PERIMAX, or unresectable metastatic CRC to be randomized between FOLFOX and bevacizumab with or without irinotecan, stratified for clinical groups according to disease and patients' characteristics in the CHARTA trial.
\end{abstract}

Trial registration: Clinical trial identifier CHARTA: NCT01321957, PERIMAX: NCT01540435

Keywords: FOLFOXIRI, Bevacizumab, Metastatic colorectal cancer, Resectable liver metastases

\footnotetext{
* Correspondence: hans-joachim.schmoll@uk-halle.de

${ }^{\dagger}$ Equal contributors

${ }^{3}$ Department for Oncology/Hematology, Martin-Luther-University Halle,

Ernst-Grube-Str. 40, 06120 Halle/Saale, Germany

Full list of author information is available at the end of the article
} 


\section{Background}

Colorectal cancer (CRC) is the most frequently diagnosed cancer in Europe and one of the leading causes of cancer death worldwide [1,2]. About $20-25 \%$ of patients with CRC present with metastatic disease at time of diagnosis, and further $20-25 \%$ of patients will develop metastases after curative resection, of whom 20-30\% present with liver metastases only [3,4]. Upfront stratification of patients with metastatic CRC (mCRC) into clinical groups according to clinical presentation and treatment aim by a multidisciplinary team is of utmost importance for the prognosis of the individual patient [5]. Clinical groups based on current guidelines and recommendations are displayed in Table 1 [6,7]. Recent retrospective series demonstrated profound improvements in outcome of patients with colorectal cancer over the last 20 years due to advancements in chemotherapy and dramatic increase in patients undergoing liver resection for either primarily or secondarily resectable colorectal liver metastases (CLM), resulting in an overall survival (OS) comparable to that of stage III (UICC) colon cancer $[8,9]$.

\section{Management of R0 resectable liver metastases}

Resection of CLM is a potentially curative treatment, and reported 5-year survival rates are about 40\% [10,11]. Further follow up after resection of liver metastases from colorectal cancer demonstrated long-term survival and finally cure for every fifth patient even in the presence of poor prognostic factors [12-15]. However, with the majority of patients relapsing after liver resection, either intrahepatic (about 70\%), extrahepatic (about 50\%), or both, further research is urgently warranted [16].

\section{Selection of patients for a potentially curative approach}

The selection of patients for hepatic surgery is a controversial issue. The most commonly used scoring system for prediction of OS described by Fong and colleagues incorporates five risk factors: node-positive primary, diseasefree interval $<12$ months, $>1$ lesion, size $>5 \mathrm{~cm}$, and CEA $>200 \mathrm{ng} / \mathrm{m}$. Patients with 5 risk factors had a 5 year OS rate (5-yOSR) of $14 \%$ with a median of 22 months compared to a 5 -yOSR of $44 \%$ and a median of 51 months in

Table $\mathbf{1}$ Clinical groups for first-line treatment
stratification
\begin{tabular}{|ll}
\hline group & clinical presentation \\
\hline 0 & clearly Ro-resectable liver and/or lung metastases \\
\hline 1 & $\begin{array}{l}\text { unresectable liver and/or lung metastasis potentially } \\
\text { resectable after downsizing, comorbidities allowing surgery }\end{array}$ \\
\hline 2 & $\begin{array}{l}\text { multiple metastasis, rapid progression, risk of rapid } \\
\text { deterioration, unlikely to become resectable }\end{array}$ \\
\hline 3 & never resectable and no symptoms or risk of deterioration
\end{tabular}

case of one risk factor [14]. Recently, the international liver metastases survey, including data of about 4500 patients with preoperatively treated CLM, confirmed the poor prognosis of patients with lymph node positive primary, abnormal CEA levels, and a high number of CLM, although cut off value was $>3$ [15].

Whereas the estimation of survival is broadly accepted, criteria for resectability are far from being well and uniformly defined [17-21]. Generally, a post-resection remnant liver of less than $30 \%$, unfavourable location of metastases, co-morbidities excluding major surgery, aggressive tumour biology, and/or presence of extrahepatic disease are regarded as limitations for liver surgery. However, liver resection should have the potential for complete macroscopic resection in curative intent [21].

\section{Perioperative treatment for resectable CLM}

The EORTC 40983 trial accrued 364 patients to be randomized to two treatment arms: surgery alone or chemotherapy with 5-FU/LV and oxaliplatin (FOLFOX4 regimen), administered 3 months pre- and postoperatively. [22]. Patients had to be technically resectable (assessed by CT scan) with a maximum of 4 CLM and no prior treatment with chemotherapy. Despite favourable patients' characteristics, with more than $50 \%$ bearing only a single CLM and about $80 \%$ with up to 2 , respectively, and performance of surgery in "high quality" institutions, the reported 3 year progression free survival rates (3-yPFSR) were relatively poor. In the group of patients $(n=171)$ receiving the planned chemotherapy and resection of CLM 3-yPFSR significantly increased from $33.2 \%$ (surgery alone) to $42.4 \%$ (HR 0.73, $95 \%$ CI 0.55 to 0.97 ). However, in the "intent to treat analyses", the difference was not statistically significant due to ineligibility of $6 \%$ of patients (HR 0.79, $95 \%$ CI 0.62 to 1.02). No unusual toxicities occurred in the chemotherapy arm, and about $80 \%$ of patients completed the preoperative part. After resection, $76 \%$ of patients received postoperative treatment with the majority $(52 \% ; \mathrm{n}=80)$ completing all 6 cycles.

Results of two single arm phase II trials using capecitabine, oxaliplatin and bevacizumab as neoadjuvant treatment in about 100 patients with CLM either clearly R0 resectable or unlikely R0 resectable with poor prognosis demonstrated feasibility of this regimen with an overall response rate (ORR) of $73-78 \%$ and a conversion rate of $40 \%$ (12 of 30) in the unlikely resectable group [23,24].

\section{Postoperative systemic treatment}

Two randomized phase III trials have compared adjuvant systemic chemotherapy with $5 \mathrm{FU} / \mathrm{LV}$ after resection of CLM to surgery alone, but both were closed prematurely due to slow accrual. By the time of closure, a small but statistically significant improvement in disease 
free survival (DFS) could be shown in the French trial, with a 5-year DFS rate of 26.7 vs. $33.5 \% \quad(p=0.028)$, favouring the group with adjuvant treatment [25]. The ENG (EORTC/NCI-CTG/GIVIO) trial, still not fully published, showed a non-significant trend towards a prolongation of DFS (median 39 vs. 20 months; $\mathrm{p}=0.35$ ) and an increase in overall survival (median 53 vs. 43 months; $\mathrm{p}=0.39$ ) [26]. The combined analysis of both trials $(\mathrm{n}=278$ patients) showed a non-significant prolongation of DFS from 18.8 to 27.9 months $(\mathrm{p}=0.058)$ and OS from 47.3 to 62.2 months $(\mathrm{p}=0.095)$ [27]. Both trials were using a (non-contemporary) 5-FU bolus regimen.

Intensification of postoperative treatment by $5-\mathrm{FU} / \mathrm{LV}$ plus irinotecan in a prematurely stopped, randomized phase III trial revealed no additional benefit compared to 5-FU/LV alone in terms of DFS (21.6 vs. 24.7 months, $\mathrm{p}=0.47$ ) and OS (3-year rate of $71.6 \mathrm{vs}$. 72.7\%, p=0.69) [28].

The Dutch HEPATICA trial, prematurely stopped after 74 randomized patients, indicated that postoperative treatment with capecitabine and oxaliplatin for 6 months and bevacizumab for 12 months might be beneficial with a trend in DFS rate at 2 years of 50 vs. $72 \%(p=0.074)$, although this trend needs to be carefully interpreted in regard of the known only transient benefit achieved with prolonged bevacizumab in stage II/III colon cancer [29-31].

\section{First line treatment for $\mathrm{mCRC}$}

Several first-line treatment options are currently available incorporating fluoropyrimidines, irinotecan, oxaliplatin, bevacizumab and EGFR antibodies (e.g. cetuximab and panitumumab) for KRAS wildtype patients [32-37].

\section{Bevacizumab based first line treatment for mCRC}

A variety of trials and registry analyses evaluated the efficacy of bevacizumab-containing first-line regimens. The addition of bevacizumab to 5FU bolus and irinotecan regimen (IFL) significantly increased ORR and prolonged OS and PFS [38]. The NO16966 study was initially designed to prove non-inferiority of the XELOX regimen compared with FOLFOX-4, but the addition of bevacizumab in a $2 \times 2$ factorial design was amended after the above-mentioned results. Although ORR in this trial was not different between the bevacizumab and the placebo arms, potentially curative surgery was performed in $55(9.6 \%)$ patients on bevacizumab vs. $38(6.6 \%)$ on placebo $(\mathrm{p}=0.061)$ in the per protocol population. PFS was significantly increased in the bevacizumab group, 9.4 vs. 8.0 months in the placebo group (HR 0.83, 97.5\% CI 0.72 to $0.95 ; \mathrm{p}=0.0023)$. However, OS was 21.3 months in the bevacizumab group and 19.9 months in the placebo group ( $\mathrm{HR} 0.89,97.5 \%$ CI 0.76 to 1.03; $\mathrm{p}=0.077$ ) [34]. Several observational phase IIIb/IV trials (e.g., First BEAT, BriTE) confirmed the safety profile of bevacizumab in first-line $\mathrm{mCRC}$ patients receiving a variety of chemotherapy regimens, namely FOLFOX, XELOX, FOLFIRI, or capecitabine $[39,40]$.

\section{Four drug regimens for $\mathrm{mCRC}$}

After the negative trials combining a chemo-doublet with bevacizumab and EGFR antibodies, four drug regimens focused on a chemo-triplet in combination with a targeted drug [41,42]. Several chemo-triplet schedules with 5FU/LV or capecitabine, irinotecan and oxaliplatin were evaluated with the Italian FOLFOXIRI demonstrating the most efficacious and reliable data [37,43-46]. The FOLFOXIRI regimen by Falcone et al. significantly increased ORR (34 vs. 60\%, p<0.0001), PFS (6.9 vs. 9.8 months, $\mathrm{p}=0.0006)$, and OS (16.7 vs. 22.6 months, $\mathrm{p}=0.032$ ) compared to FOLFIRI in a phase III trial with 244 patients. Furthermore, the FOLFOXIRI regimen resulted in an increased $\mathrm{R} 0$ secondary resection rate $(6 \%$ vs. $15 \%, \mathrm{p}=0.033$, among all 244 patients; and 12 vs. $36 \%, p=0.017$ among patients with CLM only) [37]. Administration of second line does not seem to be relevantly impaired by first line FOLFOXIRI according to recently presented data demonstrating feasibility and efficacy of second line treatment with either oxaliplatin or irinotecan based doublet (38\%), rechallenge with FOLFOXIRI (24\%) or fluoropyrimidine with or without mitomycin [47]. Retreatment with FOLFOXIRI was associated with significantly prolonged survival.

Several single arm phase II trials with different 5FU/LV, irinotecan and oxaliplatin schedules were performed in combination with either bevacizumab or cetuximab resulting in high ORR of $75-82 \%$ [48-53]. However, in the absence of comparative data, toxicity profile (especially grade $3 / 4$ diarrhoea) seems to favour bevacizumab-containing regimens (14-28\%) compared to $36-93 \%$ observed with cetuximab combinations even after dose reduction of chemotherapy.

In a recently published single arm phase II trial, an ORR of $77 \%$ in all patients and $80 \%$ in the liver-only population $(n=30)$ was achieved with the combination of FOLFOXIRI and bevacizumab. R0 resections were performed in $40 \%(n=12)$ of the liver-only population. Disease control rate was $100 \%$. PFS and OS were 13.1 and 30.9 months, respectively. Treatment was well tolerated with the major grade $3 / 4$ toxicity being neutropenia in $49 \%$ of patients (compared to $50 \%$ with FOLFOXIRI alone), which was well manageable with secondary G-CSF prophylaxis leading to only one case of febrile neutropenia. Further G3/4 toxicities were diarrhoea in $14 \%$, hypertension in $11 \%$, and asthenia as well as deep vein thrombosis in $7 \%$ of patients [52]. Similar results could be achieved in another 5FU/LV, irinotecan and oxaliplatin regimen (weekly alternating "Poker" schedule) with 
bevacizumab [49]. Furthermore, early safety results of a phase III trial by the Italian group comparing FOLFIRI and bevacizumab with or without oxaliplatin revealed no unexpected toxicities in the first 100 randomized patients [54].

\section{Methods/design Study design}

Both trials are multicentre, open labelled, prospective, randomized phase II studies. The study protocols were approved by the local ethics committees, and were also subject to authorization by the competent authority (BfArM for CHARTA and PEI for PERIMAX) as mandatory by federal law. All participants have to provide written informed consent. The trials were assigned the EudraCT numbers 2010-022162-27 for CHARTA and 2010-023575-25 for PERIMAX and are registered at ClinicalTrials.gov (CHARTA: NCT01321957, PERIMAX: NCT01540435).

\section{Study objectives and endpoints}

The primary objective of the CHARTA study is to evaluate the efficacy of FOLFOXIRI and bevacizumab compared to FOLFOX and bevacizumab in patients with initially unresectable metastatic colorectal cancer. Secondary objectives are safety and tolerability of the treatment, efficacy in terms of secondary resectability, prognostic value of stratification into clinical groups, and validity of allocation to these groups and the exploratory question whether the addition of irinotecan might be more effective in terms of response and survival in patient groups to be determined by angiogenic marker profiles, potentially indicating different sensitivity to bevacizumab. Primary endpoint is PFS rate at 9 months; secondary endpoints include PFS, OS, ORR (according to RECIST v1.1), secondary resection rate, toxicity (according to NCI-CTCAE v4.0) and quality of life (according to EORTC QLQ-C30 and modules CR29 and CIPN20) [55]. PFS is defined as time from randomization to date of first observed progression or death (without reintroduction).

The primary objective of the PERIMAX study is to evaluate the efficacy of FOLFOX for 6 months postoperatively compared to FOLFOXIRI and bevacizumab for three months pre- and three months postoperatively for primarily resectable liver metastases from colorectal cancer. Secondary objectives are safety and tolerability of the treatment as well as efficacy in terms of survival. Primary endpoint is failure- free survival (FFS) rate at 18 months. Failure will be defined as macroscopically incomplete resection (R2), local or distant recurrence or death from any cause. Secondary endpoints are PFS, FFS, OS, achievability of macroscopically complete resection (R0/1), ORR (according to RECIST v1.1) after preoperative treatment (arm B), toxicity (according to NCI-CTCAE $\mathrm{v} 4.0$ ), perioperative morbidity, quality of life (according to EORTC QLQ-C30 and module LMC21), and survival according to molecular or clinicopathological factors.

\section{Patient selection}

Patients with histologically confirmed diagnosis of mCRC can be included in the CHARTA-PERIMAX trials, independent of synchronous or metachronous metastases, although in case of synchronous disease in the PERIMAX trial the primary tumour needs to be asymptomatic and clearly $\mathrm{R} 0$ resectable. Inclusion into the PERIMAX trial is limited to patients with R0 resectable CLM as judged by the treating physician and thus excludes patients with extrahepatic disease. Measurable disease according to RECIST, adequate ECOG-PS ( $\leq 2$ in CHARTA, although ECOG-PS 2, only if tumour related; $\leq 1$ in PERIMAX), age $\geq 18$ (upper limit in the PERIMAX at 75 years of age), prior adjuvant treatment completed either at least 6 months before inclusion in the CHARTA, or at least 12 months for oxaliplatin-based treatment in the PERIMAX, are further selection criteria. Prerequisite laboratory values for both trials are absolute neutrophil counts $\geq 1.5 \times 10^{9} / \mathrm{L}$, platelets $\geq 100$ $\mathrm{x} 10^{9} / \mathrm{L}$, haemoglobin $\geq 9 \mathrm{~g} / \mathrm{dl}$ or $5.59 \mathrm{mmol} / \mathrm{l}$, INR $<1.5$ and aPTT $<1.5$ Upper Limit of Normal (ULN) for patients not receiving therapeutic anticoagulation (use of full dose anticoagulants is allowed as long as the INR or aPTT is within therapeutic limits and the patient has been on a stable dose for anticoagulants for at least two weeks at the time of registration), serum transaminases (AST \& ALT) $\leq 2.5 \times$ ULN (in case of liver metastases $<5 \times$ ULN), total bilirubin $\leq 1.5 \mathrm{x}$ ULN, and creatinine $\leq 1.5 \mathrm{x}$ ULN. Further selection criteria are based on current standard criteria (e.g. no pregnancy or breast feeding, signed and dated consent form) and the contraindications of the used agents (e.g. severe thrombosis or bleeding, major surgery within 28 days, chronic diarrhoea or significant peripheral neuropathy).

\section{Treatment schedule}

Treatment schedules for CHARTA and PERIMAX are summarized in Figures 1 and 2, respectively. Patients will randomly be assigned in a 1:1 ratio stratified for the clinical groups one to three mentioned in Table 1 in the CHARTA trial and for number of CLM $\leq 3$ vs $>3$ and Fong score $<2$ vs. $\geq 2$ in the PERIMAX trial.

\section{CHARTA trial}

Induction chemotherapy with a modified FOLFOX with oxaliplatin at a dose of $85 \mathrm{mg} / \mathrm{m}^{2}$ iv over two hours (day 1 ), LV at a dose of $200 \mathrm{mg} / \mathrm{m}^{2}$ iv over two hours (day 1) and 5 -FU at a dose of $3200 \mathrm{mg} / \mathrm{m}^{2}$ iv over 


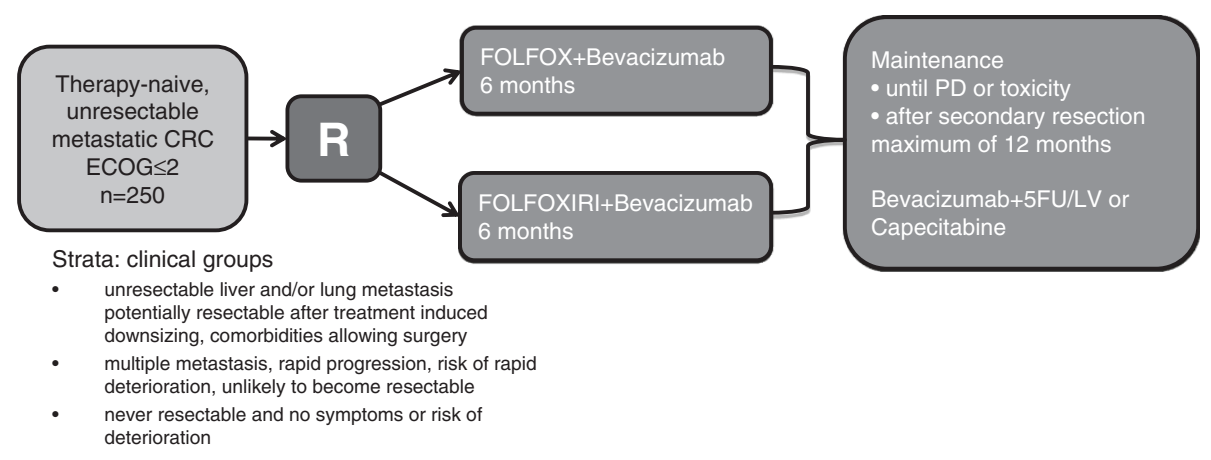

Figure 1 overview CHARTA trial.

48 hours (day 1-3) and bevacizumab at a dose of $5 \mathrm{mg} / \mathrm{kg}$ iv over 30 to $90 \mathrm{~min}$ (day 1) with or without irinotecan at a dose of $165 \mathrm{mg} / \mathrm{m}^{2}$ iv over one hour (day 1 ) in a biweekly schedule will be administered followed by maintenance with either 5-FU/LV and bevacizumab (same dosage and schedule as above) or capecitabine at a dose of $1600 \mathrm{mg} / \mathrm{m}^{2}$ in two doses po day 1 to 14 and bevacizumab at a dose of $7.5 \mathrm{mg} / \mathrm{kg}$ iv over 30 to $90 \mathrm{~min}$ (day 1 ) every three weeks (choice of 5-FU or capecitabine is at the discretion of the investigator). Treatment with FOLFOX and bevacizumab $+/$ - irinotecan will be administered until progression, intolerable toxicity, and secondary resection or for a maximum of 12 cycles ( 6 months). After 6 months of treatment and/or no progression patients will continue with a maintenance regimen with bevacizumab and a fluoropyrimidine for up to 12 months in the absence of progression or intolerable toxicity. Maximum treatment duration is 18 months (6 months of FOLFOX and bevacizumab +/- irinotecan followed by 12 months of maintenance). In case of secondary resection (at any time point) treatment should be resumed 4-8 weeks postoperatively for a total of 6 months FOLFOX and bevacizumab +/irinotecan (pre- and postoperative treatment), followed by maintenance treatment for total treatment duration of up to 12 months in the absence of progression or intolerable toxicity. Reintroduction of oxaliplatin $+/-$ irinotecan or restart of treatment in case of progressive disease during maintenance or complete break after 18 months of treatment is at the investigators discretion.

\section{PERIMAX trial}

The same regimens as in the CHARTA trial will be administered, although the postoperative treatment in arm $\mathrm{A}$ is FOLFOX without bevacizumab. In arm $\mathrm{A}$ FOLFOX will be administered for 12 cycles (6 months) postoperatively starting $6+/-2$ weeks after surgery. In the perioperative arm (B) FOLFOXIRI and bevacizumab will be administered for 6 cycles (last cycle without bevacizumab) (3 months) preoperatively, followed by liver surgery within $6+/-2$ weeks after last treatment FOLFOXIRI (without bevacizumab), followed by 6 cycles ( 3 months) $6+/-2$ weeks postoperatively. Bevacizumab should be withheld for at least 5 weeks before conducting surgery.

The first cycle of FOLFOXIRI in both trials can be administered with $75 \%$ of dosage, if no diarrhoea or other significant toxicities $\geq$ grade 2 occurs, following cycles should be administered in full dosage. Dose reduction and escalation is at the discretion of the investigator. Preoperative dose modifications should be maintained for postoperative treatment in the PERIMAX trial. If a patient was treated with full dose preoperatively, dose reduction to $75 \%$ for the first cycle and subsequent escalation might be applied similar to preoperative treatment. Doses of drugs will be reduced in

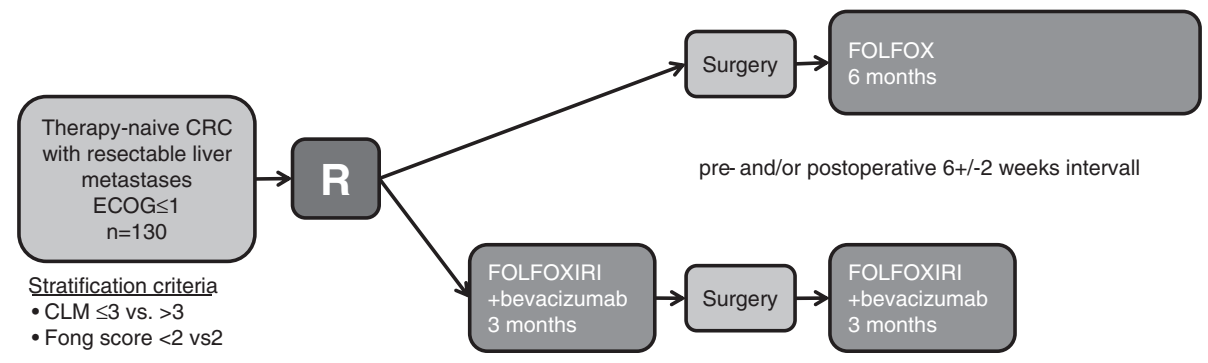

Figure 2 overview PERIMAX trial. 
case of undue toxicity. Treatment will be delayed until adequate recovery from toxicity.

\section{Assessments/follow up}

Tolerability will be evaluated at every visit including physical examination, vital signs, clinical laboratory profile, and adverse events, which will be graded according to NCI-CTCAE v.4.0 and correlated to the administered treatment or performed study procedure. Treatment related Serious Adverse Events (SAE) considered possibly, probably or definitely related to treatment, will be determined.

In both trials the investigator will assess tumour response according to RECIST v1.1 and CEA (optional CA 19-9). CT and/or MRI scans will be independently reviewed e.g. for resectability (both trials) and allocation to the clinical groups (CHARTA). In the CHARTA trial imaging of all sites of disease will be performed at baseline and every 4 cycles ( 8 weeks) thereafter for the first 6 months (induction treatment) and afterwards every 3 months (during and after maintenance) until progression.

Radiological imaging in the PERIMAX trial (CT-scan of the chest, CT or MRI-scan of the abdomen) should be done at baseline and/or preoperatively, postoperatively (prior to the start of postoperative treatment) and afterwards every three months for two years and every 6 month thereafter in both arms. In the preoperatively treated arm, an additional radiological imaging after the $3^{\text {rd }}$ cycle (6 weeks) should be done to avoid clinically complete response. In case of CEA elevation without progressive disease defined by RECIST, further examinations must be performed searching for local recurrence or metastatic progression of disease. Diagnosis of recurrence could either be made by radiological imaging or by positive cytology or biopsy. After progression, patients will be followed every 3 months for disease status, protracted toxicities, further treatment, and survival.

Quality of life will be assessed together with tumour assessment using the EORTC QLQ-C30, and the modules CR29 and CIPN20 in the CHARTA and the module LMC21 in the PERIMAX.

Formalin fixed and paraffin embedded (FFPE) tumour tissue obtained at baseline (if available) and serum obtained at baseline and before the $3^{\text {rd }}$ and the $7^{\text {th }}$ cycle will be evaluated for KRAS and BRAF-status (FFPE) and angiogenic markers (FFPE and serum) in the CHARTA trial. In addition to KRAS and BRAF in PERIMAX evaluation of pathohistological response rate and further analyses are planned, thus either paraffin embedded or preferably fresh frozen tissue of primary tumour and hepatic metastases should be obtained in patients consenting. Furthermore, serum obtained at baseline, during preoperative treatment, postoperatively and at follow up will be evaluated for circulating tumour DNA.

\section{Statistical considerations and sample size estimation CHARTA trial}

First line therapy with FOLFOX and bevacizumab displays a median PFS of 9,4 months [34], leading to a PFS Rate at 9 months (PFSR@9) of approximately 55\%. The four-drug combination treatment should produce a PFSR@9 of at least $71.3 \%$. The risk of estimating the four-drug combination treatment as active although the PFSR@9 is less than $71.3 \%$ should be $10 \%$. The risk of rejecting the therapy although the PFSR@9 is more than 71.3\% should be 20\%, which leads to a power of $80 \%$. The planned number of patients to answer this question in a two-sided (continuity corrected) Chi-square test will be 120 and with a dropout rate of $4 \%$ the number to be included will be 125 patients per arm. Analysis will be performed by logistic regression to account for the stratification.

\section{PERIMAX trial}

Perioperative chemotherapy with 5-FU/LV with or without oxaliplatin in a prognostic favourable group of patients with up to 4 CLM led to a FFS rate at 18 months (FFSR@18) of 50-65\% [22,25]. However, in the trial by Portier et al.. solely patients after R0 resection were included, whereas in the EORTC study 51\% and $27 \%$ of patients had one or two CLM, respectively. In this trial, patients with unlimited number of CLM, fulfilling the local judgement for resectability will be included. Therefore,FFSR@18 is expected to be 40\% with current treatment strategies (postoperative FOLFOX). The investigational treatment (perioperative FOLFOXIRI and bevacizumab) should produce a FFSR@18 of at least 60\%, to be regarded as promising for further evaluation and of major clinical relevance. The trial should achieve $80 \%$ power to detect differences between the treatments and keeping the type I error level below $10 \%$ in a one-sided (continuity corrected) Chi-square test. The planned number of patients to answer this question with a drop out rate of about $14 \%$ will be 65 patients per arm. The trial uses a randomized parallel arm phase II design.

\section{Quality assurance/safety}

Patient data are collected in a case report form at the data centre of the clinical research organisation (Koordinierungszentrum für Klinische Studien Halle). Consistency checks will be performed on newly entered forms and queries issued in case of inconsistencies. Onsite monitoring will be performed adapted according to the site accrual. Data safety monitoring boards will review the data from both trials on a regular basis.

\section{Discussion}

The CHARTA and PERIMAX trials are designed to evaluate the benefits and limitations of a highly active four-drug regimen in different treatment situations of 
metastatic CRC with nearly 200 patients randomized to an experimental treatment with FOLFOXIRI and bevacizumab vs. what is considered a standard approach.

Perioperative treatment in resectable CLM, especially its sequence (peri- vs. postoperative treatment) and intensity, is still a matter of debate. The PERIMAX trial design represents the agreement on a multidisciplinary trial by surgical and medical oncologists in Germany and reflects the ongoing discussion about timing of perioperative chemotherapy. In regard of the high recurrence rate after resection with curative intent and the results of four drug combinations including bevacizumab in $\mathrm{mCRC}$, further intensification of the perioperative treatment to improve patients' prognosis by increasing the rate of long-term survivors seems to be a feasible option. Despite the lack of data for FOLFOX for purely postoperative treatment after resection of CLM, this regimen was chosen in regard of the beneficial trend for $5 \mathrm{FU} / \mathrm{LV}$ in this setting and the data obtained in stage III disease with oxaliplatin [27,56,57]. Furthermore, this treatment approach is widely used for patients receiving upfront resection of CLM, and is considered a 'current standard' for this patient group. However, the PERIMAX trial has several variables (sequence and intensity) and is therefore of an exploratory nature focusing on the efficacy and feasibility of perioperative FOLFOXIRI and bevacizumab in this setting. The randomized design was chosen to collect data albeit limited by the low number of patients on the comparative efficacy in terms of survival in order to reduce the bias that is associated with a comparison to historical controls, which are only available for 5FU/LV in this setting [58]. Number of CLM with a cut-off value of 3 was added to the widely accepted stratification according to the Fong score. Although already part of the Fong score, this cut off value has recently demonstrated high prognostic value [15]. The composite endpoint FFS was chosen to adapt to the specific situation in this trial with half of the patients being disease-free within the first months after randomization, compared to the other half receiving 3 months of preoperative treatment. In order to ensure comparability with available data, e.g. EORTC 40983 (PFS), and to gain information about tolerability and QoL with the four-drug regimen secondary endpoints include these parameters. Perioperative treatment was limited to 6 months, as no conclusive data about prolonged treatment is currently available [30]. The trial also offers the unique opportunity of collecting tissue from liver metastases and primary tumours either untreated or treated. Therefore, fresh frozen and paraffin embedded tissue will be collected and stored for further analyses.

The CHARTA trial will evaluate the efficacy of a maximal intensive first line regimen compared to a standard first line regimen in patients with unresectable metastatic
CRC. The intensification of first line treatment is believed to increase the rate of patients amenable for a secondary curative approach and even if surgery might not be an option this treatment approach could result in improved long-term disease control. Especially for clinical group 2 and 3, if amenable for intensive first line treatment, disease control after induction chemotherapy could result in long intervals of either low toxic maintenance therapy or complete treatment discontinuation and might thus be a reasonable long-term approach. Furthermore, the magnitude and point in time of tumour response has demonstrated significant impact on prognosis, underlining the need for an intensive first line regimen inducing early tumour shrinkage [59]. Eligible patients will be stratified according to clinical groups in order to balance the arms especially for potentially secondary resectable patients. PFS-rate at 9 months was chosen as primary endpoint to gain information about the comparative efficacy in terms of a survival endpoint, taking into account the inclusion of all metastatic and not only potentially resectable patients. Treatment duration is limited to a maximum of 6 months FOLFOX and bevacizumab +/- irinotecan, followed by up to 12 months of maintenance with fluoropyrimidine and bevacizumab. According to current clinical standard oxaliplatin should be discontinued at least after 6 months of treatment due to significant cumulative side effects, particularly peripheral neuropathy. None of the available trials with FOLFOXIRI with or without bevacizumab continued treatment beyond 12 cycles (6 months), as tolerability will be significantly impaired and achievement of secondary resectability with prolonged treatment beyond 6 months seems unlikely [37,52]. In the CELIM trial for example median time to resection or exploration was 5.1 months, with a median number of 8 treatment cycles until intervention [60]. Current data support the use of maintenance treatment after first line chemotherapy, though the choice of regimen is not yet clear [61-64]. However, results of ongoing trials (e.g. AIO 0207 and CAIRO3) might help clarifying this question and potentially the choice of maintenance regimen as well (biological +/- fluoropyrimidine. In regard of the higher intensity of the four-drug combination not only in terms of response but as well in terms of toxicity QoL evaluation is performed during the trial.

With overall 380 previously untreated metastatic CRC patients classified for resectability, stratified for clinical grouping and randomized, the CHARTA and PERIMAX trials will give further information about the benefit and tolerability of FOLFOXIRI and bevacizumab in these distinct disease settings.

\section{Abbreviations}

5-FU, 5-fluorouracil; 5-yOSR, 5 year overall survival rate; $A E$, Adverse event; ALT, Alanin-aminotransferase; AST, Aspartat-aminotransferase; aPTT, Activated 
partial thromboplastin time; BfArM, Bundesinstitut für Arzneimittel und Medizinprodukte; BRAF, V-raf murine sarcoma viral oncogene homolog B1; CA 19-9, Carbohydrate antigen 19-9; CEA, Carcinoembryonic antigen; CLM, Colorectal liver metastases; CT, Computed tomography; DFS, Disease free survival; DNA, Deoxyribonucleic acid; ECOG-PS, Eastern cooperative oncology group - performance status; EGFR, Epidermal growth factor receptor; EORTC, European organisation for research and treatment of cancer; EudraCT, European Clinical Trials Database; FFS, Failure free survival; FFSR@18, Failure free survival rate at 18 months; FOLFIRI, 5-FU/LV and irinotecan; FOLFOX, 5-FU/LV and oxaliplatin; FOLFOXIRI, 5-FU/LV, oxaliplatin and irinotecan; G, Grade; G-CSF, Granulocyte colony-stimulating factor; HR, Hazard ratio; IFL, 5FU bolus and irinotecan regimen; INR, International Normalized Ratio; IV, Intravenous; KRAS, Kirsten rat sarcoma viral oncogene homolog; LV, Leucovorin; mCRC, Metastatic colorectal cancer; min, Minutes; $\mathrm{MRI}$, Magnetic resonance imaging; N, Number of patients; $\mathrm{NCl}$ -

CTCAE, National Cancer Institute common terminology criteria for adverse events; ORR, Overall response rate; OS, Overall survival; P, P-value; PEI, Paul Ehrlich Institut; PFS, Progression free survival; PFSR@9, Progression free survival rate at 9 months; Po, Per os; RECIST, Response evaluation criteria in solid tumours; SAE, Severe adverse event; QoL, Quality of life; QLQ, Quality of life questionnaire; UICC, Union internationale contre le cancer; ULN, Upper Limit of Normal; V, Version; Vs, Versus; XELOX, Capecitabine and oxaliplatin.

\section{Competing interests}

Both trials are funded by Roche. AS has received honoraria from Roche and Merck KGaA. HJS has received research funding and honoraria from Roche. $\mathrm{H}-\mathrm{JS}$ and DA have received honoraria and research funding from Roche and Merck KGaA and honoraria from Amgen. BH has received honoraria and research funding from Amgen, Roche and Merck KGaA. AW, AK, GG, MR and TE declare that there is no conflict of interest.

\section{Authors' contributions}

AS and GG prepared the manuscript. HJS and H-JS are the coordinating investigators for the two trials and participated in the preparation of manuscript and study protocols. AS wrote both study protocols, GG participated in the PERIMAX protocol. DA coordinates the translational part of the CHARTA trial. AW performed the statistical analysis for both trials and participated in drafting of the manuscript. MR coordinates both trials as clinical research associate. $\mathrm{TE}, \mathrm{BH}, \mathrm{SH}, \mathrm{Gl}, \mathrm{AK}$ participate in patient recruitment in both trials. All authors read and approved the final manuscript.

\section{Author details \\ ${ }^{1}$ University Cancer Center Hamburg, University Medical Center Hamburg-Eppendorf, Hamburg, Germany. ${ }^{2}$ Department of Surgery, University Medical Center Regensburg, Regensburg, Germany. ${ }^{3}$ Department for Oncology/Hematology, Martin-Luther-University Halle, Ernst-Grube-Str. 40, 06120 Halle/Saale, Germany. ${ }^{4}$ MedCenter Nordsachsen, Schkeuditz, Germany. ${ }^{5}$ Charité Centrum für Tumormedizin, Campus Virchow-Klinikum, Berlin, Germany. ${ }^{6}$ Department for Gastroenterology, Academic Teaching Hospital, Celle, Germany. ${ }^{7}$ Department of Hematology/Oncology, Comprehensive Cancer Center University of Freiburg, Freiburg, Germany. ${ }^{8}$ Department of Surgery, University Hospital of Tübingen, Tübingen, Germany. \\ ${ }^{9}$ Koordinierungszentrum Klinische Studien Halle, Martin-Luther-University, Halle, Germany.}

Received: 11 April 2012 Accepted: 17 July 2012

Published: 16 August 2012

\section{References}

1. Jemal A, Bray F, Center MM, Ferlay J, Ward E, Forman D: Global cancer statistics. CA Cancer J Clin 2011, 61:69-90.

2. Ferlay J, Shin HR, Bray F, Forman D, Mathers C, Parkin DM: Estimates of worldwide burden of cancer in 2008: GLOBOCAN 2008. Int J Cancer 2010, 127(12):2893-2917.

3. van der Pool AE, Damhuis RA, ljzermans JN, de Wilt JH, Eggermont AM, Kranse R, Verhoef C: Trends in incidence, treatment and survival of patients with stage IV colorectal cancer: a population-based series. Colorectal Dis 2012, 14:56-61.

4. Mantke R, Schmidt U, Wolff S, Kube R, Lippert H: Incidence of synchronous liver metastases in patients with colorectal cancer in relationship to clinico-pathologic characteristics. Results of a German prospective multicentre observational study. Eur J Surg Oncol 2012, 38:259-265.

5. Schmoll HJ, Sargent D: Single agent fluorouracil for first-line treatment of advanced colorectal cancer as standard? Lancet 2007, 370:105-107.

6. Van Cutsem E, Dicato M, Arber N, Berlin J, Cervantes A, Ciardiello F, De Gramont A, Diaz-Rubio E, Ducreux M, Geva R, et al: Molecular markers and biological targeted therapies in metastatic colorectal cancer: expert opinion and recommendations derived from the 11th ESMO/World Congress on Gastrointestinal Cancer, Barcelona, 2009. Ann Oncol 2010, 21(Suppl 6):vi1-10.

7. Schmiegel W, Pox C, Arnold D, Porschen R, Rodel C, Reinacher-Schick A: Colorectal carcinoma: the management of polyps, (neo)adjuvant therapy, and the treatment of metastases. Dtsch Arztebl Int 2009, 106:843-848.

8. Kopetz S, Chang GJ, Overman MJ, Eng C, Sargent DJ, Larson DW, Grothey A, Vauthey JN, Nagorney DM, McWilliams RR: Improved survival in metastatic colorectal cancer is associated with adoption of hepatic resection and improved chemotherapy. J Clin Oncol 2009, 27:3677-3683.

9. Morris EJ, Forman D, Thomas JD, Quirke P, Taylor EF, Fairley L, Cottier B, Poston G: Surgical management and outcomes of colorectal cancer liver metastases. Br J Surg 2010, 97:1110-1118.

10. Jonas S, Thelen A, Benckert C, Spinelli A, Sammain S, Neumann U, Rudolph B, Neuhaus P: Extended resections of liver metastases from colorectal cancer. World J Surg 2007, 31:511-521.

11. Wei AC, Greig PD, Grant D, Taylor B, Langer B, Gallinger S: Survival after hepatic resection for colorectal metastases: a 10-year experience. Ann Surg Oncol 2006, 13:668-676.

12. Tomlinson JS, Jarnagin WR, DeMatteo RP, Fong Y, Kornprat P, Gonen M, Kemeny N, Brennan MF, Blumgart LH, D'Angelica M: Actual 10-year survival after resection of colorectal liver metastases defines cure. J Clin Oncol 2007, 25:4575-4580.

13. Scheele J, Stang R, Altendorf-Hofmann A, Paul M: Resection of colorectal liver metastases. World J Surg 1995, 19:59-71.

14. Fong Y, Fortner J, Sun RL, Brennan MF, Blumgart LH: Clinical score for predicting recurrence after hepatic resection for metastatic colorectal cancer: analysis of 1001 consecutive cases. Ann Surg 1999, 230:309-318. discussion 318-321.

15. Adam R, Barroso E: Impact of the type and modalities of preoperative chemotherapy on the outcome of liver resection for colorectal metastases. J Clin Oncol 2011, 29:abstr 3519.

16. de Jong MC, Pulitano C, Ribero D, Strub J, Mentha G, Schulick RD, Choti MA, Aldrighetti L, Capussotti L, Pawlik TM: Rates and patterns of recurrence following curative intent surgery for colorectal liver metastasis: an international multi-institutional analysis of 1669 patients. Ann Surg 2009, 250:440-448.

17. Nagashima I, Takada T, Nagawa H, Muto T, Okinaga K: Proposal of a new and simple staging system of colorectal liver metastasis. World J Gastroenterol 2006, 12:6961-6965.

18. Poston GJ, Figueras J, Giuliante F, Nuzzo G, Sobrero AF, Gigot JF, Nordlinger B, Adam R, Gruenberger T, Choti MA, et al: Urgent need for a new staging system in advanced colorectal cancer. J Clin Oncol 2008, 26:4828-4833.

19. Van Cutsem E, Nordlinger B, Adam R, Kohne CH, Pozzo C, Poston G, Ychou M, Rougier P: Towards a pan-European consensus on the treatment of patients with colorectal liver metastases. Eur J Cancer 2006, 42:2212-2221.

20. Yamaguchi T, Mori T, Takahashi K, Matsumoto H, Miyamoto H, Kato T: A new classification system for liver metastases from colorectal cancer in Japanese multicenter analysis. Hepatogastroenterology 2008, 55:173-178.

21. Nordlinger B, Van Cutsem E, Gruenberger T, Glimelius B, Poston G, Rougier $\mathrm{P}$, Sobrero A, Ychou M: Combination of surgery and chemotherapy and the role of targeted agents in the treatment of patients with colorectal liver metastases: recommendations from an expert panel. Ann Oncol 2009, 20:985-992.

22. Nordlinger B, Sorbye H, Glimelius B, Poston GJ, Schlag PM, Rougier P, Bechstein WO, Primrose JN, Walpole ET, Finch-Jones $\mathrm{M}$, et al: Perioperative chemotherapy with FOLFOX4 and surgery versus surgery alone for resectable liver metastases from colorectal cancer (EORTC Intergroup trial 40983): a randomised controlled trial. Lancet 2008, 371:1007-1016.

23. Wong R, Cunningham D, Barbachano Y, Saffery C, Valle J, Hickish T, Mudan S, Brown G, Khan A, Wotherspoon A, et al: A multicentre study of 
capecitabine, oxaliplatin plus bevacizumab as perioperative treatment of patients with poor-risk colorectal liver-only metastases not selected for upfront resection. Ann Oncol 2011, 22:2042-2048.

24. Gruenberger B, Tamandl D, Schueller J, Scheithauer W, Zielinski C, Herbst F, Gruenberger T: Bevacizumab, capecitabine, and oxaliplatin as neoadjuvant therapy for patients with potentially curable metastatic colorectal cancer. J Clin Oncol 2008, 26:1830-1835.

25. Portier G, Elias D, Bouche O, Rougier P, Bosset JF, Saric J, Belghiti J, Piedbois P, Guimbaud R, Nordlinger $B$, et al: Multicenter randomized trial of adjuvant fluorouracil and folinic acid compared with surgery alone after resection of colorectal liver metastases: FFCD ACHBTH AURC 9002 trial. J Clin Oncol 2006, 24:4976-4982.

26. Langer B, Bleiberg H, Labianca R, et al: Fluorouracil (FU) plus I-leucovorin (I-LV) versus observation after potentially curative resection of liver or lung metastases from colorectal cancer (CRC): results of the ENG (EORTC/NCIC CTG/GIVIO) randomized trial. Proc Am Soc Clin Oncol 2002, 21(abstr 592):2002.

27. Mitry E, Fields AL, Bleiberg H, Labianca R, Portier G, Tu D, Nitti D, Torri V, Elias D, O'Callaghan C, et al: Adjuvant chemotherapy after potentially curative resection of metastases from colorectal cancer: a pooled analysis of two randomized trials. J Clin Oncol 2008, 26:4906-4911.

28. Ychou M, Hohenberger W, Thezenas S, Navarro M, Maurel J, Bokemeyer C, Shacham-Shmueli E, Rivera F, Kwok-Keung Choi C, Santoro A: A randomized phase III study comparing adjuvant 5-fluorouracil/folinic acid with FOLFIRI in patients following complete resection of liver metastases from colorectal cancer. Ann Oncol 2009, 20:1964-1970.

29. Andre T, Van Cutsem E: A multinational, randomized phase III study of bevacizumab (Bev) with FOLFOX4 or XELOX versus FOLFOX4 alone as adjuvant treatment for colon cancer (CC): Subgroup analyses from the AVANT trial. J Clin Oncol 2011, 29:abstr 3509.

30. Voest EE, Snoeren N: A randomized two-arm phase III study to investigate bevacizumab in combination with capecitabine plus oxaliplatin (CAPOX) versus CAPOX alone in post radical resection of patients with liver metastases of colorectal cancer. J Clin Oncol 2011, 29:abstr 3565.

31. Allegra CJ, Yothers G, O'Connell MJ, Sharif S, Petrelli NJ, Colangelo LH, Atkins JN, Seay TE, Fehrenbacher L, Goldberg RM, et al: Phase III trial assessing bevacizumab in stages II and III carcinoma of the colon: results of NSABP protocol C-08. J Clin Oncol 2011, 29:11-16.

32. Douillard JY, Siena S, Cassidy J, Tabernero J, Burkes R, Barugel M, Humblet Y, Bodoky G, Cunningham D, Jassem J, et al: Randomized, phase III trial of panitumumab with infusional fluorouracil, leucovorin, and oxaliplatin (FOLFOX4) versus FOLFOX4 alone as first-line treatment in patients with previously untreated metastatic colorectal cancer: the PRIME study. $J$ Clin Oncol 2010, 28:4697-4705.

33. Van Cutsem E, Kohne CH, Lang I, Folprecht G, Nowacki MP, Cascinu S, Shchepotin I, Maurel J, Cunningham D, Tejpar S, et al: Cetuximab Plus Irinotecan, Fluorouracil, and Leucovorin As First-Line Treatment for Metastatic Colorectal Cancer: Updated Analysis of Overall Survival According to Tumor KRAS and BRAF Mutation Status. J Clin Oncol 2011, 29:2011-2019.

34. Saltz LB, Clarke S, Diaz-Rubio E, Scheithauer W, Figer A, Wong R, Koski S, Lichinitser M, Yang TS, Rivera F, et al: Bevacizumab in combination with oxaliplatin-based chemotherapy as first-line therapy in metastatic colorectal cancer: a randomized phase III study. J Clin Oncol 2008, 26:2013-2019

35. Maughan TS, Adams RA, Smith CG, Meade AM, Seymour MT, Wilson RH, Idziaszczyk S, Harris R, Fisher D, Kenny SL, et al: Addition of cetuximab to oxaliplatin-based first-line combination chemotherapy for treatment of advanced colorectal cancer: results of the randomised phase 3 MRC COIN trial. Lancet 2011, 377:2103-2114.

36. Bokemeyer C, Bondarenko I, Hartmann JT, de Braud F, Schuch G, Zubel A, Celik I, Schlichting M, Koralewski P: Efficacy according to biomarker status of cetuximab plus FOLFOX-4 as first-line treatment for metastatic colorectal cancer: the OPUS study. Ann Oncol 2011, 22:1535-1546.

37. Falcone A, Ricci S, Brunetti I, Pfanner E, Allegrini G, Barbara C, Crino L, Benedetti G, Evangelista W, Fanchini $L$, et al: Phase III trial of infusional fluorouracil, leucovorin, oxaliplatin, and irinotecan (FOLFOXIRI) compared with infusional fluorouracil, leucovorin, and irinotecan (FOLFIRI) as first-line treatment for metastatic colorectal cancer: the Gruppo Oncologico Nord Ovest. J Clin Oncol 2007, 25:1670-1676.
38. Hurwitz H, Fehrenbacher L, Novotny W, Cartwright T, Hainsworth J, Heim W, Berlin J, Baron A, Griffing S, Holmgren E, et al: Bevacizumab plus irinotecan, fluorouracil, and leucovorin for metastatic colorectal cancer. N Engl J Med 2004, 350:2335-2342.

39. Van Cutsem E, Rivera F, Berry S, Kretzschmar A, Michael M, DiBartolomeo M, Mazier MA, Canon $J \mathrm{~L}$, Georgoulias V, Peeters M, et al: Safety and efficacy of first-line bevacizumab with FOLFOX, XELOX, FOLFIRI and fluoropyrimidines in metastatic colorectal cancer: the BEAT study. Ann Oncol 2009, 20:1842-1847.

40. Grothey A, Sugrue MM, Purdie DM, Dong W, Sargent D, Hedrick E, Kozloff $\mathrm{M}$ : Bevacizumab beyond first progression is associated with prolonged overall survival in metastatic colorectal cancer: results from a large observational cohort study (BRiTE). J Clin Oncol 2008, 26:5326-5334.

41. Hecht JR, Mitchell E, Chidiac T, Scroggin C, Hagenstad C, Spigel D, Marshall J, Cohn A, McCollum D, Stella P, et al: A randomized phase IIIB trial of chemotherapy, bevacizumab, and panitumumab compared with chemotherapy and bevacizumab alone for metastatic colorectal cancer. J Clin Oncol 2009, 27:672-680.

42. Tol J, Koopman M, Cats A, Rodenburg CJ, Creemers GJ, Schrama JG, Erdkamp FL, Vos AH, van Groeningen CJ, Sinnige HA, et al: Chemotherapy, bevacizumab, and cetuximab in metastatic colorectal cancer. $N$ Engl J Med 2009, 360:563-572.

43. Bajetta E, Celio L, Ferrario E, Di Bartolomeo M, Denaro A, Dotti K, Mancin M, Bajetta R, Colombo A, Pusceddu S: Capecitabine plus oxaliplatin and irinotecan regimen every other week: a phase I/II study in first-line treatment of metastatic colorectal cancer. Ann Oncol 2007, 18:1810-1816.

44. Ychou M, Viret F, Kramar A, Desseigne F, Mitry E, Guimbaud R, Delpero JR, Rivoire M, Quenet F, Portier G, Nordlinger B: Tritherapy with fluorouracil/ leucovorin, irinotecan and oxaliplatin (FOLFIRINOX): a phase II study in colorectal cancer patients with non-resectable liver metastases. Cancer Chemother Pharmacol 2008, 62:195-201.

45. Souglakos J, Androulakis N, Syrigos K, Polyzos A, Ziras N, Athanasiadis A, Kakolyris S, Tsousis S, Kouroussis C, Vamvakas L, et al: FOLFOXIRI (folinic acid, 5-fluorouracil, oxaliplatin and irinotecan) vs FOLFIRI (folinic acid, 5-fluorouracil and irinotecan) as first-line treatment in metastatic colorectal cancer (MCC): a multicentre randomised phase III trial from the Hellenic Oncology Research Group (HORG). Br J Cancer 2006, 94:798-805

46. Zarate R, Rodriguez J, Bandres E, Patino-Garcia A, Ponz-Sarvise M, Viudez A, Ramirez N, Bitarte N, Chopitea A, Gacia-Foncillas J: Oxaliplatin, irinotecan and capecitabine as first-line therapy in metastatic colorectal cancer (mCRC): a dose-finding study and pharmacogenomic analysis. $\mathrm{Br} J$ Cancer 2010, 102:987-994.

47. Fornaro L, Vasile E, Masi G, Loupakis F, Baldi GG, Allegrini G, Salvatore L, Cremolini C, Cupini S, Cortesi E, et al: Outcome of Second-Line Treatment After First-Line Chemotherapy With the GONO FOLFOXIRI Regimen. Clin Colorectal Cancer 2012, 11:71-76.

48. Assenat E, Desseigne F, Thezenas S, Viret F, Mineur L, Kramar A, Samalin E, Portales F, Bibeau F, Crapez-Lopez E, et al: Cetuximab Plus FOLFIRINOX (ERBIRINOX) as first-line treatment for unresectable metastatic colorectal cancer: a phase II trial. Oncologist 2011, 16(11):1557-1564.

49. Bruera G, Santomaggio A, Cannita K, Baldi PL, Tudini M, De Galitiis F, Mancini M, Marchetti P, Antonucci A, Ficorella C, Ricevuto E: "Poker" association of weekly alternating 5 -fluorouracil, irinotecan, bevacizumab and oxaliplatin (FIr-B/FOx) in first line treatment of metastatic colorectal cancer: a phase II study. BMC Cancer 2010, 10:567.

50. Trarbach T, Schuette J: Dose escalating study of 5-FU/folinic acid (FA)/ oxaliplatin/irinotecan (FOLFOXIRI) and cetuximab in first-line therapy of patients with metastatic colorectal cancer. J Clin Oncol 2009, 27:abstr e15025.

51. Ychou M, Desseigne F: Preliminary results of a multicentre phase II trial evaluating cetuximab in combination with FOLFIRINOX (LV5FU + Irinotecan + Oxaliplatin) as first line treatment of metastatic colorectal cancer (mCRC) patients. Gastrointestinal Cancers Symposium 2009, 2009 ((Abstract-No: 295):abstr. 450.

52. Masi G, Loupakis F, Salvatore L, Fornaro L, Cremolini C, Cupini S, Ciarlo A, Del Monte F, Cortesi E, Amoroso D, et al: Bevacizumab with FOLFOXIRI (irinotecan, oxaliplatin, fluorouracil, and folinate) as first-line treatment for metastatic colorectal cancer: a phase 2 trial. Lancet Oncol 2010, 11:845-852. 
53. Garufi C, Torsello A, Tumolo S, Ettorre GM, Zeuli M, Campanella C, Vennarecci G, Mottolese M, Sperduti I, Cognetti F: Cetuximab plus chronomodulated irinotecan, 5-fluorouracil, leucovorin and oxaliplatin as neoadjuvant chemotherapy in colorectal liver metastases: POCHER trial. Br J Cancer 2010, 103:1542-1547.

54. Falcone A, Loupakis F: FOLFOXIRI plus bevacizumab (BV) versus FOLFIRI plus $B V$ as first-line treatment of metastatic colorectal cancer (MCRC): Preliminary safety results of the phase III randomized TRIBE study by the Gruppo Oncologico Nord-Ovest (GONO). J Clin Oncol 2010, 28:abstr 3543.

55. Eisenhauer EA, Therasse P, Bogaerts J, Schwartz LH, Sargent D, Ford R, Dancey J, Arbuck S, Gwyther S, Mooney M, et al: New response evaluation criteria in solid tumours: revised RECIST guideline (version 1.1). Eur $J$ Cancer 2009, 45:228-247.

56. Andre T, Boni C, Navarro M, Tabernero J, Hickish T, Topham C, Bonetti A, Clingan P, Bridgewater J, Rivera F, de Gramont A: Improved overall survival with oxaliplatin, fluorouracil, and leucovorin as adjuvant treatment in stage II or III colon cancer in the MOSAIC trial. J Clin Oncol 2009, 27:3109-3116.

57. Haller DG, Tabernero J, Maroun J, de Braud F, Price T, Van Cutsem E, Hill M, Gilberg F, Rittweger K, Schmoll HJ: Capecitabine Plus Oxaliplatin Compared With Fluorouracil and Folinic Acid As Adjuvant Therapy for Stage III Colon Cancer. J Clin Oncol 2011, 29:1465-1471.

58. Ratain MJ, Sargent DJ: Optimising the design of phase II oncology trials: the importance of randomisation. Eur J Cancer 2009, 45:275-280.

59. Piessevaux H, Schlichting M: Early tumor shrinkage for the prediction of efficacy of cetuximab in metastatic colorectal cancer: analysis from the CRYSTAL study. Ann Oncol 2010, 21:abstr. 596.

60. Folprecht G, Gruenberger T, Bechstein WO, Raab HR, Lordick F, Hartmann JT, Lang H, Frilling A, Stoehlmacher J, Weitz J, et al: Tumour response and secondary resectability of colorectal liver metastases following neoadjuvant chemotherapy with cetuximab: the CELIM randomised phase 2 trial. Lancet Oncol 2010, 11:38-47.

61. Wasan H, Adams RA: Intermittent chemotherapy (CT) plus continuous or intermittent cetuximab $(C)$ in the first-line treatment of advanced colorectal cancer (aCRC): Results of the two-arm phase II randomized MRC COIN-b trial. J Clin Oncol 2012, 30:abstr 536.

62. Adams RA, Meade AM, Seymour MT, Wilson RH, Madi A, Fisher D, Kenny SL, Kay E, Hodgkinson E, Pope M, et al: Intermittent versus continuous oxaliplatin and fluoropyrimidine combination chemotherapy for first-line treatment of advanced colorectal cancer: results of the randomised phase 3 MRC COIN trial. Lancet Oncol 2011, 12:642-653.

63. Chibaudel B, Maindrault-Goebel F, Lledo G, Mineur L, Andre T, Bennamoun M, Mabro M, Artru P, Carola E, Flesch M, et al: Can chemotherapy be discontinued in unresectable metastatic colorectal cancer? The GERCOR OPTIMOX2 Study. J Clin Oncol 2009, 27:5727-5733.

64. Diaz-Rubio E, Gomez-Espana A, Massuti B, Sastre J, Abad A, Valladares M Rivera F, Safont MJ, Martinez de Prado P, Gallen M, et al: First-Line XELOX Plus Bevacizumab Followed by XELOX Plus Bevacizumab or Single-Agent Bevacizumab as Maintenance Therapy in Patients with Metastatic Colorectal Cancer: The Phase III MACRO TTD Study. Oncologist 2012, 17:15-25.

doi:10.1186/1471-2407-12-356

Cite this article as: Stein et al:: Treatment with bevacizumab and FOLFOXIRI in patients with advanced colorectal cancer: presentation of two novel trials (CHARTA and PERIMAX) and review of the literature. BMC Cancer 2012 12:356.

\section{Submit your next manuscript to BioMed Central and take full advantage of:}

- Convenient online submission

- Thorough peer review

- No space constraints or color figure charges

- Immediate publication on acceptance

- Inclusion in PubMed, CAS, Scopus and Google Scholar

- Research which is freely available for redistribution 